\title{
On the analyticity of functions approximated by their q-Bernstein polynomials when $q>1$
}

\author{
Iossif Ostrovskii ${ }^{\mathrm{a}}$, Sofiya Ostrovska ${ }^{\mathrm{b}, *}$ \\ a Bilkent University, Department of Mathematics, 06800 Bilkent, Ankara, Turkey \\ ${ }^{\mathrm{b}}$ Atilim University, Department of Mathematics, 06836 Incek, Ankara, Turkey
}

\section{A R T I C L E I N F O}

\section{Keywords:}

$q$-Integers

$q$-Bernstein polynomials

Uniform convergence

Analytic function

Analytic continuation

\begin{abstract}
A B S T R A C T
Since in the case $q>1$ the $q$-Bernstein polynomials $B_{n, q}$ are not positive linear operators on $C[0,1]$, the investigation of their convergence properties for $q>1$ turns out to be much harder than the one for $0<q<1$. What is more, the fast increase of the norms $\left\|B_{n, q}\right\|$ as $n \rightarrow \infty$, along with the sign oscillations of the $q$-Bernstein basic polynomials when $q>1$, create a serious obstacle for the numerical experiments with the $q$-Bernstein polynomials.

Despite the intensive research conducted in the area lately, the class of functions which are uniformly approximated by their $q$-Bernstein polynomials on $[0,1]$ is yet to be described. In this paper, we prove that if $f:[0,1] \rightarrow \mathbb{C}$ is analytic at 0 and can be uniformly approximated by its $q$-Bernstein polynomials $(q>1)$ on $[0,1]$, then $f$ admits an analytic continuation from $[0,1]$ into $\{z:|z|<1\}$.
\end{abstract}

(ㄷ) 2010 Elsevier Inc. All rights reserved.

\section{Introduction}

The importance of the Bernstein polynomials opened the gates to the discovery of their numerous generalizations as well as their applications in various mathematical disciplines, see, for example, [1-8]. Due to the speedy development of the $q$ calculus, recent generalizations based on the $q$-integers have emerged. Lupaş was the person who pioneered the work on the $q$-versions of the Bernstein polynomials. In 1987, he introduced (cf. [9]) a $q$-analogue of the Bernstein operator, and investigated its approximation and shape-preserving properties (see also [10]).

Recently another generalization, called the q-Bernstein polynomials, has been brought into the spotlight and studied by a number of authors from different perspectives. A review of the results on the $q$-Bernstein polynomials, along with an extensive bibliography on this subject and a collection of open problems is given in [11]. The subject remains under ample study, and there have been new papers constantly coming out (see, for example, [12-14] published after [11]).

To present our results, let us recall the necessary notations and definitions. Let $q>0$. For any $n \in \mathbb{Z}_{+}$, the $q$-integer $[n]_{q}$ is defined by:

$$
[n]_{q}:=1+q+\cdots+q^{n-1}(n \in \mathbb{N}), \quad[0]_{q}:=0
$$

and the $q$-factorial $[n]_{q}$ ! by:

$$
[n]_{q} !:=[1]_{q}[2]_{q} \ldots[n]_{q}(n=1,2, \ldots), \quad[0]_{q} !:=1 .
$$

For integers $0 \leqslant k \leqslant n$, the $q$-binomial coefficient is defined by:

\footnotetext{
* Corresponding author.

E-mail addresses: iossif@fen.bilkent.edu.tr (I. Ostrovskii), ostrovskasofiya@yahoo.com, ostrovsk@atilim.edu.tr (S. Ostrovska).
} 


$$
\left[\begin{array}{l}
n \\
k
\end{array}\right]_{q}:=\frac{[n]_{q} !}{[k]_{q} ![n-k]_{q} !}
$$

Definition 1.1. Let $f:[0,1] \rightarrow \mathbb{C}$. The $q$-Bernstein polynomials of $f$ are:

$$
B_{n, q}(f ; z)=\sum_{k=0}^{n} f\left(\frac{[k]_{q}}{[n]_{q}}\right) p_{n k}(q ; z), \quad n \in \mathbb{N},
$$

where

$$
p_{n k}(q ; z):=\left[\begin{array}{l}
n \\
k
\end{array}\right]_{q} z^{k} \prod_{j=0}^{n-k-1}\left(1-q^{j} z\right), \quad k=0,1, \ldots n
$$

are the $q$-Bernstein basic polynomials.

Note that for $q=1$, we recover the classical Bernstein polynomials. We reserve the name " $q$-Bernstein polynomials" for the new polynomials appearing when $q \neq 1$.

It has been known (cf. [11] and references therein) that some properties of the classical Bernstein polynomials are extended to the $q$-Bernstein polynomials. For example, the $q$-Bernstein polynomials possess the end-point interpolation property, the shape-preserving properties in the case $0<q<1$, and representation via divided differences. Like the Bernstein polynomials, the $q$-Bernstein polynomials reproduce linear functions, and are degree-reducing on the set of polynomials.

On the other hand, the approximation properties of the $q$-Bernstein polynomials are essentially different from those of the classical ones. What is more, the cases $0<q<1$ and $q>1$ are not similar to each other. This absence of similarity is caused by the fact that, for $0<q<1, B_{n, q}$ are positive linear operators on $C[0,1]$, while for $q>1$, the positivity does not hold any longer. It should be pointed out that in terms of the convergence properties, the similarity between the classical Bernstein and $q$-Bernstein polynomials ceases to be true even in the case $0<q<1$, see, e.g., [15,16]. This is because, for $0<q<1$, the $q$-Bernstein polynomials -despite being positive linear operators - do not satisfy the conditions of Korovkin's Theorem. They do, however, satisfy the conditions of Wang's Korovkin-type theorem (cf. [17]), serving as a model example for the theorem.

Due to the lack of positivity, the study of the convergence properties of the $q$-Bernstein polynomials in the case $q>1$ turns out to be essentially more complicated than the one in the case $0<q<1$. In spite of the intensive research conducted in this area recently, the class of functions in $C[0,1]$ uniformly approximated by their $q$-Bernstein polynomials when $q>1$ is yet to be described. However, the results obtained for specific classes of functions have already revealed some new phenomena as well as interesting facts (see, e.g., $[12,13,18]$ ). To some extent, the explanation for such an 'exotic' behaviour of the $q$-Bernstein polynomials is presented in [14]. It has been proved there that basic polynomials (1.1) combine the fast increase in magnitude (namely, $\left.\left\|p_{n k}\right\|_{[0,1]} \asymp \frac{1}{n} q^{(n+k+1)(n-k) / 2}, n \rightarrow \infty\right)$ with the sign oscillations on [0,1]. This creates substantial hurdles in the numerical study of the $q$-Bernstein polynomials for $q>1$.

It is exactly this unexpected behavior of $q$-Bernstein polynomials with respect to convergence that makes the study of such properties interesting and challenging.

In this paper, we present new results on the convergence of the $q$-Bernstein polynomials. It has been known (see [19], Theorem 1) that entire functions and, in particular, polynomials are uniformly approximated by their $q$-Bernstein polynomials $(q>1)$ on any compact set in $\mathbb{C}$. The aim of this paper is to examine the properties of functions allowing the uniform approximation by the $q$-Bernstein polynomials. It will be proved that if $f:[0,1] \rightarrow \mathbb{C}$ is analytic at 0 and can be uniformly approximated by its $q$-Bernstein polynomials $(q>1)$ on $[0,1]$, then $f$ admits an analytic continuation from $[0,1]$ into $\{z$ : $|z|<1\}$. Let it be emphasized that this is the first result giving a necessary condition for the approximation in the case $q>1$.

\section{Statement of results}

In the sequel, we denote by $D_{a}$ and by $\bar{D}_{a}$ an open and closed disc, respectively, of radius a centered at 0 .

It has been known (see [20]) that if a function $f$ is bounded on $[0,1]$ and admits an analytic continuation $f(z)$ from $[0, a)$ to $D_{a}$, then

$$
B_{n, q}(f ; z) \rightarrow f(z) \text { as } n \rightarrow \infty,
$$

uniformly on any compact set in $D_{a}$.

Examples given in $[12,18]$ show that outside of $D_{a}$ the uniform approximation may be impossible on any interval.

In this paper, we present some general statements on the approximation of analytic functions by their $q$-Bernstein polynomials.

We assume throughout the paper that $q>1$ is fixed. Our main result is the following:

Theorem 2.1. Let $f \in C[0,1]$ admit an analytic continuation into $D_{a}, 0<a<1$. If there exist $c, d \in \mathbb{R}$ so that $d>c>a$ and the sequence $\left\{B_{n, q}(f ; x)\right\}$ is uniformly bounded on an interval $[c, d]$, then $f(x)$ admits an analytic continuation $\tilde{f}(z)$ from $[0, a)$ into some disc $D_{b}$ with $b>a$. 
Remark 2.1. In general $\tilde{f}(x) \neq f(x)$ for $a<x<b$.

As an immediate application of this theorem, we obtain the following results.

Corollary 2.1. Let $f(x) \in C[0,1]$ and admit an analytic continuation into a disc $D_{a}, 0<a<1$. If $f(x)$ does not admit an analytic continuation from $[0, a)$ into a disc $D_{b}, b>a$, then $f(x)$ cannot be uniformly approximated by its $q$-Bernstein polynomials on $[0,1]$.

Theorem 2.2. Let $f(z)$ be analytic at 0 , so that $f(x) \in C[0,1]$. If

$$
B_{n, q}(f ; x) \rightarrow f(x) \text { as } n \rightarrow \infty,
$$

uniformly on $[0,1]$, then $f(x)$ admits an analytic continuation into $D_{1}$.

Remark 2.2. In general, $f(z)$ may not be continuous in $\bar{D}_{1}$. For example, $f(x)=1 / \sqrt{1+x}$ is uniformly approximated by $B_{n}$, ${ }_{q}(f ; x)$ on $[0,1]$, as it has been proved in [12], Corollary 2.7 .

\section{Some auxiliary results}

In this section, we present a series of facts required to prove Theorems 2.1 and 2.2. The section contains some new statements as well as previously available ones, which have been included for the purpose of convenience of the readers.

Let

$$
B_{n, q}(f ; z)=\sum_{k=0}^{n} c_{k n} z^{k}, \quad n \in \mathbb{N},
$$

be the $q$-Bernstein polynomials of $f$. We use the following representation of these polynomials given in [19], formulae (6) and (7):

$$
B_{n, q}(f ; z)=\sum_{k=0}^{n} \lambda_{k n} f\left[0 ; \frac{1}{[n]_{q}} ; \ldots ; \frac{[k]_{q}}{[n]_{q}}\right] z^{k},
$$

where $f\left[x_{0} ; x_{1} ; \ldots ; x_{k}\right]$ denotes the divided differences of $f$, that is

$$
\begin{aligned}
& f\left[x_{0}\right]=f\left(x_{0}\right), \quad f\left[x_{0}, x_{1}\right]=\frac{f\left(x_{1}\right)-f\left(x_{0}\right)}{x_{1}-x_{0}}, \ldots, \\
& f\left[x_{0} ; x_{1} ; \ldots ; x_{k}\right]=\frac{f\left[x_{1} ; \ldots ; x_{k}\right]-f\left[x_{0} ; \ldots ; x_{k-1}\right]}{x_{k}-x_{0}}
\end{aligned}
$$

and $\lambda_{k n}$ are given by

$$
\lambda_{0 n}=\lambda_{1 n}=1, \quad \lambda_{k n}=\prod_{j=1}^{k-1}\left(1-\frac{[j]_{q}}{[n]_{q}}\right), \quad k=2, \ldots n .
$$

Remark 3.1. It has been shown in [19] that $\lambda_{k n}(k=0,1, \ldots, n)$ are eigenvalues of the $q$-Bernstein operator $B_{n, q}$. For $q=1$, we recover eigenvalues of the Bernstein operator, whose eigenstructure is described in [21].

If $f$ is an analytic function, then (cf. e.g., [22], Section 2.7, p. 44) the divided differences of $f$ can be expressed by:

$$
f\left[x_{0} ; x_{1} ; \ldots ; x_{k}\right]=\frac{1}{2 \pi i} \oint_{\mathscr{L}} \frac{f(\zeta) d \zeta}{\left(\zeta-x_{0}\right) \ldots\left(\zeta-x_{k}\right)},
$$

where $\mathscr{L}$ is a contour encircling $x_{0}, \ldots, x_{k}$ and $f$ is assumed to be analytic on and within $\mathscr{L}$.

We need the following property of the eigenvalues (3.2).

Lemma 3.1. Let $q>1$. Then for all $k, n$, we have:

$$
\lambda_{k n}=: \lambda_{k n}^{(q)} \geqslant \prod_{j=1}^{\infty}\left(1-q^{-j}\right)=: \lambda_{q}>0 .
$$

Proof. For $k=0,1$, the statement is obvious. Let $k>1$. Using the simple inequality:

$$
\frac{[n-j]_{q}}{[n]_{q}}<\frac{1}{q^{j}} \text { for } q>1
$$


we obtain

$$
\lambda_{k n}^{(q)}=\prod_{j=1}^{k-1}\left(1-\frac{[j]_{q}}{[n]_{q}}\right) \geqslant \prod_{j=1}^{n-1}\left(1-\frac{[j]_{q}}{[n]_{q}}\right)=\prod_{j=1}^{n-1}\left(1-\frac{[n-j]_{q}}{[n]_{q}}\right) \geqslant \prod_{j=1}^{n-1}\left(1-\frac{1}{q^{j}}\right) \geqslant \prod_{j=1}^{\infty}\left(1-\frac{1}{q^{j}}\right)=\lambda_{q} .
$$

In the case of $f(x)$ admitting an analytic continuation from $[0,1]$ to a disc centered at 0 , the coefficients of $B_{n, q}(f ; \cdot)$ can be estimated with the help of the following lemma.

Lemma 3.2 [20]. Let $f(x) \in C[0,1]$ and possess an analytic continuation into a closed disc $\overline{D_{a}}$. If

$$
B_{n, q}(f ; z)=\sum_{k=0}^{n} c_{k n} z^{k}
$$

then the following estimate holds:

$$
\left|c_{k n}\right| \leqslant \frac{C_{a f}}{a^{k}},
$$

where $C_{a, f}$ is independent from both $k$ and $n$.

The reasonings of the present paper have been based largely upon the following statement.

Lemma 3.3. Let $q>1$. Given $0 \neq \zeta \in \mathbb{C}$, we fix $m \in \mathbb{N}, \varepsilon>0$ in such a way that

$$
|\zeta|>q^{-m}+\varepsilon
$$

Then for all $n>m$, the following estimate holds:

$$
\left|\prod_{s=m}^{n-1}\left(1-\frac{[n-s]_{q}}{\zeta[n]_{q}}\right)-\prod_{s=m}^{\infty}\left(1-\frac{1}{\zeta q^{s}}\right)\right| \leqslant C n q^{-n},
$$

where $C=C_{q, \varepsilon}$ is independent from both $n$ and $m$.

Corollary 3.1. For any $0 \neq \zeta \in \mathbb{C}, k \in \mathbb{N}$, we have:

$$
\lim _{n \rightarrow \infty} \prod_{s=k}^{n-1}\left(1-\frac{[n-s]_{q}}{\zeta[n]_{q}}\right)=\prod_{s=k}^{\infty}\left(1-\frac{1}{\zeta q^{s}}\right) .
$$

Proof of Lemma 3.3. We denote:

$$
\Delta_{m n}:=\prod_{s=m}^{n-1}\left(1-\frac{[n-s]_{q}}{\zeta[n]_{q}}\right)-\prod_{s=m}^{\infty}\left(1-\frac{1}{\zeta q^{s}}\right)=: I_{m n}-I_{m, \infty} .
$$

Consider

$$
\ln I_{m n}-\ln I_{m, \infty}=\sum_{s=m}^{n-1}\left(\ln \left(1-\frac{[n-s]_{q}}{\zeta[n]_{q}}\right)-\ln \left(1-\frac{1}{\zeta q^{s}}\right)\right)-\sum_{s=n}^{\infty} \ln \left(1-\frac{1}{\zeta q^{s}}\right)=: S_{m n}+\sigma_{n} .
$$

Let us estimate $\sigma_{n}$ first. Indeed, by virtue of (3.5), we have:

$$
\left|\frac{1}{\zeta q^{s}}\right|<1 \text { for } s \geqslant m,
$$

whence

$$
\left|\sigma_{n}\right| \leqslant \sum_{s=n}^{\infty}\left|\ln \left(1-\frac{1}{\zeta q^{s}}\right)\right| \leqslant \sum_{s=n}^{\infty} \frac{1 /\left(|\zeta| q^{s}\right)}{1-1 /\left(|\zeta| q^{s}\right)} \leqslant \frac{1}{1-1 /\left(|\zeta| q^{n}\right)} \cdot \frac{1}{|\zeta|} \cdot \sum_{s=n}^{\infty} q^{-s}=\frac{1}{|\zeta|-q^{-n}} \cdot \frac{q}{(q-1) q^{n}} .
$$

Since $|\zeta|-q^{-n} \geqslant|\zeta|-q^{-m}>\varepsilon$, we obtain:

$$
\left|\sigma_{n}\right| \leqslant \frac{1}{\varepsilon} \cdot \frac{q}{(q-1) q^{n}}=: \frac{C_{1}}{q^{n}},
$$

where $C_{1}$ depends only on $q$ and $\varepsilon$.

Now, we estimate $S_{m n}$ :

$$
\left|S_{m n}\right| \leqslant \sum_{s=m}^{n-1}\left|\ln \left(1-\frac{[n-s]_{q}}{\zeta[n]_{q}}\right)-\ln \left(1-\frac{1}{\zeta q^{s}}\right)\right|=\sum_{s=m}^{n-1}\left|\int_{1 /\left(\zeta q^{s}\right)}^{[n-s]_{q} /\left(\zeta[n]_{q}\right)} \frac{d z}{1-z}\right|,
$$


where the integral is taken along the straight line segment joining its limits. We notice that

$$
\left|\frac{n-s]_{q}}{\zeta[n]_{q}}\right|<\frac{1}{|\zeta| q^{s}} \leqslant \frac{1}{|\zeta| q^{m}} \leqslant \frac{1}{1+q^{m} \varepsilon} \leqslant \frac{1}{1+\varepsilon} .
$$

Therefore, the segment of integration is contained in the disc $\overline{D_{1 /(1+\varepsilon)}}$ and its distance from $z=1$ is at least $\varepsilon /(1+\varepsilon)$. Hence, we obtain:

$$
\begin{aligned}
\left|s_{m n}\right| & \leqslant \sum_{s=m}^{n-1} \frac{1+\varepsilon}{\varepsilon} \cdot\left|\frac{[n-s]_{q}}{\zeta[n]_{q}}-\frac{1}{\zeta q^{s}}\right| \leqslant \frac{1+\varepsilon}{\varepsilon} \cdot \frac{1}{|\zeta|} \cdot \sum_{s=m}^{n-1}\left(\frac{1}{q^{s}}-\frac{[n-s]_{q}}{[n]_{q}}\right) \leqslant \frac{1+\varepsilon}{\varepsilon} \cdot \frac{1}{|\zeta|} \cdot \sum_{s=m}^{n-1} \frac{1}{q^{n}-1} \leqslant \frac{1+\varepsilon}{\varepsilon} \cdot \frac{q^{m}}{1+\varepsilon q^{m}} \cdot \frac{n}{q^{n}-1} \\
& \leqslant \frac{1+\varepsilon}{\varepsilon^{2}} \cdot \frac{n}{q^{n}-1} \leqslant C_{2} n q^{-n},
\end{aligned}
$$

where $C_{2}=C_{q, \varepsilon}$.

As a result, we obtain:

$$
\left|S_{m n}\right|+\left|\sigma_{n}\right| \leqslant C_{1} q^{-n}+C_{2} n q^{-n} \leqslant C_{3} n q^{-n},
$$

where $C_{3}$ is independent from $n$ and $k$.

Now, we estimate $\left|\Delta_{m n}\right|$ as follows:

$$
\begin{aligned}
\left|\Delta_{m n}\right| & \leqslant\left|e^{\ln I_{m n}}-e^{\ln I_{m, \infty}}\right|=\left|e^{\ln I_{m, \infty}}\right| \cdot\left|e^{\ln I_{m n}-\ln I_{m, \infty}}-1\right| \leqslant\left|I_{m, \infty}\right| \cdot e^{\left|\ln I_{m n}-\ln I_{m, \infty}\right|} \cdot\left|\ln I_{m n}-\ln I_{m, \infty}\right| \\
& \leqslant\left|\prod_{s=m}^{\infty}\left(1-\frac{1}{\zeta q^{s}}\right)\right| \cdot e^{C n q^{-n}} \cdot C n q^{-n} \leqslant \prod_{s=m}^{\infty}\left(1+\frac{1}{|\zeta| q^{s}}\right) \cdot e^{C_{3}} \cdot C_{3} n q^{-n} \leqslant C_{4} n q^{-n} \cdot \prod_{s=0}^{\infty}\left(1+\frac{1}{(1+\varepsilon) q^{s}}\right)=: C_{5} n q^{-n},
\end{aligned}
$$

with $C_{5}=C_{q, \varepsilon}$ as stated.

\section{Proofs of the theorems}

Proof of Theorem 2.1. We fix $d_{1} \in(c, d)$. Let $\tilde{\omega}$ be a harmonic measure of the interval $\left[a / d, a / d_{1}\right]$ with respect to the domain $D_{1} \backslash\left[a / d, a / d_{1}\right]$. We set:

$$
\mu:=\min _{|z|=1 / 2} \tilde{\omega}(z)>0 .
$$

Denote $\eta:=\max \left\{(a / c)^{\mu}, 1 / q\right\}$. Clearly, $\eta<1$. Now, we choose a number $a^{\prime}$ satisfying the following conditions:

(i) $a \cdot\left(c / d_{1}\right) \leqslant a^{\prime}<a$;

(ii) $a \eta<a^{\prime}<a$;

(iii) $a^{\prime} \notin\left\{q^{-j}\right\}_{j=0}^{\infty}$.

Let

$$
B_{n, q}(f ; z)=\sum_{k=0}^{n} c_{k n} z^{k}
$$

Then by Lemma 3.2, we have:

$$
\left|C_{n-k, n}\right| \leqslant \frac{C_{1}}{\left(a^{\prime}\right)^{n-k}}
$$

where $C_{1}=C_{f, q, a}$.

Assume that

$$
\left|B_{n, q}(f ; x)\right| \leqslant M \text { for } x \in[c, d] \text { with } M>1 .
$$

Consider the auxiliary polynomials:

$$
\operatorname{Pn}(z):=B_{n, q}\left(f ; a^{\prime} z\right) ;
$$

and

$$
Q_{n}(z):=z^{n} P_{n}(1 / z) .
$$

Condition (4.3) implies that

$$
\left|P_{n}(z)\right| \leqslant M\left(\frac{a^{\prime}}{c}\right)^{n} \quad \text { for } z \in\left[\frac{c}{a^{\prime}}, \frac{d}{a^{\prime}}\right],
$$


while

$$
\left|Q_{n}(z)\right| \leqslant M \cdot\left(\frac{a^{\prime}}{c}\right)^{n} \quad \text { for } z \in\left[\frac{a^{\prime}}{d}, \frac{a^{\prime}}{c}\right],
$$

In addition, for $|z| \leqslant 1$, we have by virtue of (4.2):

$$
\left|Q_{n}(z)\right| \leqslant M \cdot \sum_{k=0}^{n}\left|C_{n-k, n}\left(a^{\prime}\right)^{n-k}\right| \leqslant C_{1} n,
$$

Now, denote by $\omega_{a^{\prime}}$ the harmonic measure of the interval $\left[a^{\prime} / d, a^{\prime} / c\right]$ with respect to the domain $D_{1} \backslash\left[a^{\prime} / d, a^{\prime} / c\right]$.

Since $\left[a / d, a / d_{1}\right] \subset\left[a^{\prime} / d, a^{\prime} / c\right]$, it follows that $\omega_{a^{\prime}} \geqslant \tilde{\omega}$, that is

$$
\min _{|z|=1 / 2} \omega_{a^{\prime}} \geqslant \mu
$$

where $\mu$ is given by (4.1). To estimate $Q_{n}(z)$ in the unit disc, we apply the Two Constants Theorem (cf. e.g., [23], p. 41). It follows that, for $|z| \leqslant 1$,

$$
\left|Q_{n}(z)\right| \leqslant\left[M\left(a^{\prime} / c\right)^{n}\right]^{\omega_{a^{\prime}}(z) \cdot} \cdot\left[(n+1) C_{1}\right]^{1-\omega_{a^{\prime}}(z)} \leqslant M \cdot\left(\frac{a^{\prime}}{c}\right)^{n \omega_{a^{\prime}}(z)} \cdot\left[(n+1) C_{1}\right]^{1-\omega_{a^{\prime}}(z)} .
$$

Therefore,

$$
\max _{|z|=1 / 2}\left|Q_{n}(z)\right| \leqslant M \cdot\left(\frac{a^{\prime}}{c}\right)^{n} \cdot(n+1)^{1-\mu} \cdot C_{2} \leqslant M\left(\frac{a}{c}\right)^{\mu n} \text { for } n \geqslant n_{0} .
$$

The Cauchy estimates imply

$$
\left|C_{n-k, n}\left(a^{\prime}\right)^{n-k}\right| \leqslant 2^{k} \max _{|z|=1 / 2}\left|Q_{n}(z)\right| \leqslant C_{k, f}\left(\frac{a}{c}\right)^{\mu n} \text { for } n \geqslant n_{0} .
$$

We fix $m \in \mathbb{N}$ and $\varepsilon>0$ so that

$$
q^{-m}+\varepsilon<a^{\prime}<q^{-(m-1)}
$$

and we set:

$$
d_{m}(\zeta)=\prod_{s=m}^{\infty}\left(1-\frac{1}{\zeta q^{s}}\right)
$$

Let us estimate the integral:

$$
J:=\frac{\lambda_{n-m, n}\left(a^{\prime}\right)^{n-m}}{2 \pi i} \oint_{|z|=a^{\prime}} \frac{f(\zeta) d \zeta}{\zeta^{n-m+1} d_{m}(\zeta)} .
$$

To do this, we write using (3.1) and (3.3):

$$
C_{n-m, n}\left(a^{\prime}\right)^{n-m}=\lambda_{n-m, n} \cdot \frac{\left(a^{\prime}\right)^{n-m}}{2 \pi i} \oint_{|\zeta|=a^{\prime}} \frac{f(\zeta) d \zeta}{\zeta^{n-m+1}\left(1-\frac{1}{\zeta[n]_{q}}\right) \ldots\left(1-\frac{[n-m] q}{\zeta[n]_{q}}\right)}
$$

and obtain:

$$
J=C_{n-m, n}\left(a^{\prime}\right)^{n-m}-I,
$$

where

$$
I:=\lambda_{n-m, n} \cdot \frac{\left(a^{\prime}\right)^{n-m}}{2 \pi i} \oint_{|\zeta|=a^{\prime}} \frac{f(\zeta)}{\zeta^{n-m+1}}\left[\frac{1}{\left(1-\frac{1}{\zeta[n]_{q}}\right) \ldots\left(1-\frac{[n-m]_{q}}{\zeta[n]_{q}}\right)}-\frac{1}{d_{m}(\zeta)}\right] d \zeta .
$$

Consider the case $n>m$, where $m$ is fixed by the condition (4.5). We have:

$$
|I| \leqslant \lambda_{n-m, n} \cdot M\left(f ; a^{\prime}\right) \cdot \max _{|\zeta|=a^{\prime}}\left|\frac{d_{m}(\zeta)-\left(1-\frac{1}{\zeta[n]_{q}}\right) \ldots\left(1-\frac{[n-m]_{q}}{\zeta[n]_{q}}\right)}{\left(1-\frac{1}{\zeta[n]_{q}}\right) \ldots\left(1-\frac{[n-m]_{q}}{\zeta[n]_{q}}\right) \cdot d_{m}(\zeta)}\right| .
$$

By virtue of Lemma 3.3, we conclude that:

$$
\max _{|\zeta|=a^{\prime}}\left|d_{m}(\zeta)-\left(1-\frac{1}{\zeta[n]_{q}}\right) \cdots\left(1-\frac{[n-m]_{q}}{\zeta[n]_{q}}\right)\right| \leqslant C n q^{-n} .
$$


Now, we estimate the denominator from below as follows:

$$
\left|d_{m}(\zeta)\right|_{|\zeta|=a^{\prime}} \geqslant \prod_{j=m}^{\infty}\left(1-\frac{1}{a^{\prime} \cdot q^{j}}\right)=: C .
$$

Furthermore,

$$
\left|\left(1-\frac{1}{\zeta[n]_{q}}\right) \ldots\left(1-\frac{[n-m]_{q}}{\zeta[n]_{q}}\right)\right| \geqslant\left|d_{m}(\zeta)\right|-\left|d_{m}(\zeta)-\left(1-\frac{1}{\zeta[n]_{q}} \ldots\left(1-\frac{[n-m]_{q}}{\zeta[n]_{q}}\right)\right)\right||\geqslant| C_{1}-C_{n q}^{-n} \mid \geqslant \frac{1}{2} C_{1}
$$

for $n$ large enough.

Therefore, for $n$ large enough, we obtain:

$$
|I| \leqslant \lambda_{n-m, n} \cdot M\left(f ; a^{\prime}\right) \cdot n q^{-n}
$$

Applying (4.4) and (4.7), we obtain the following estimate:

$$
|J| \leqslant\left|C_{n-m, n}\left(a^{\prime}\right)^{n-m}\right|+|I| \leqslant C \cdot\left(\frac{a}{c}\right)^{\mu n}+C n q^{-n}
$$

for $n$ large enough.

Consider the function:

$$
F(z):=\frac{f(z) \cdot z^{m}}{d_{m}(z)}
$$

The function is analytic in $\left\{z: q^{-m}<z<a\right\}$. To prove the theorem, it suffices to show that $F$ admits an analytic continuation into $\left\{z: q^{-m}<z<b\right\}$, where $b>a$. Let

$$
F(z)=\sum_{j=-\infty}^{\infty} p_{j} z^{j}
$$

be a Laurent expansion for $F(z)$. The coefficients $\{p\}_{j=-\infty}^{\infty}$ are given by:

$$
p_{j}=\frac{1}{2 \pi i} \oint_{|\zeta|=a^{\prime}} \frac{F(\zeta) d \zeta}{\zeta^{j+1}}=\frac{1}{2 \pi i} \oint_{|\zeta|=a^{\prime}} \frac{f(\zeta) d \zeta}{\zeta^{j-m+1} d_{m}(\zeta)} .
$$

This implies for $n>m$,

$$
p_{n}=\frac{J}{\lambda_{n-m, n}\left(a^{\prime}\right)^{n-m}}
$$

Using estimates (3.4) and (4.8), we obtain:

$$
\left|p_{n}\right| \leqslant \frac{1}{\lambda_{q}} \cdot \frac{1}{\left(a^{\prime}\right)^{n-m}}\left\{C\left(\frac{a}{c}\right)^{\mu n}+C n q^{-n}\right\}
$$

for $n$ large enough.

The outer radius of convergence for series (4.9) can be estimated with the Cauchy-Hadamard formula as follows:

$$
\frac{1}{R}=\limsup _{n \rightarrow \infty} \sqrt[n]{\left|p_{n}\right|} \leqslant \frac{1}{a^{\prime}} \limsup _{n \rightarrow \infty} \sqrt[n]{C\left[\left(\frac{a}{c}\right)^{\mu n}+n q^{-n}\right]} \leqslant \frac{1}{a^{\prime}} \limsup _{n \rightarrow \infty} \sqrt[n]{2 \max \left\{\left(\frac{a}{c}\right)^{\mu}, \frac{1}{q}\right\}}=\frac{\eta}{a^{\prime}}<\frac{1}{a},
$$

by the condition (ii) for our choice of $a^{\prime}$.

Proof of Theorem 2.2. Let $0 \neq a$ : $\max \left\{r: f(x)\right.$ has an analytic continuation from $[0,1]$ into $\left.D_{r}\right\}$. We assume that $a<1$, that is

$$
q^{-(m+1)} \leqslant a<q^{-m} \text { for some } m \in \mathbb{Z}_{+} .
$$

Since the sequence $\left\{B_{n, q}(f ; x)\right\}$ is uniformly bounded on $[0,1]$, we conclude by Theorem 2.1 that there is an analytic continuation $\tilde{f}(z)$ from $[0, a)$ into a closed $\operatorname{disc} \bar{D}_{b}$ with $a<b<q^{-m}$. Let us choose $g(x) \in \mathrm{C}[0,1]$ in such a way that

$$
g(x)= \begin{cases}\tilde{f}(x) & \text { for } x \in[0, b], \\ f(x) & \text { for } x \in\left[q^{-m}-\varepsilon, 1\right]\end{cases}
$$

where $\varepsilon<\left(q^{-m}-b\right) / 2$. 
Obviously, $g(x)=f(x)$ for $x \in[0, a)$. Besides, $B_{n, q}(f ; z)=B_{n, q}(g ; z)$ for $n$ large enough, because $f$ and $g$ coincide in all of the nodes $[k]_{q} /[n]_{q}$ for $n$ large enough. By the condition of this theorem, $B_{n, q}(f(x) \rightarrow f(x)$ as $n \rightarrow \infty$ uniformly on $[0,1]$. On the other hand, it follows from [20], Theorem 2.2 that $B_{n, q}(g ; x) \rightarrow g(x)$ as $n \rightarrow \infty$ uniformly on [0,b]. This implies $f(x)=g(x)$ for $x \in[0, b]$ and, as a result, we conclude that $f(x)$ admits an analytic continuation from $[0, a)$ into $D_{b}$ with $b>a$. This contradicts our choice of $a$. Thus, $a \geqslant 1$.

\section{Acknowledgement}

We would like to express our sincere gratitude to Mr. P. Danesh from the Atilim University Academic Writing and Advisory Centre for his help in the preparation of this paper.

\section{References}

[1] J.-D. Cao, A generalization of the Bernstein polynomials, J. Math. Anal. Appl. 209 (1) (1997) 140-146.

[2] R. DeVore, G.G. Lorentz, Constructive Approximation, Springer-Verlag, 1993.

[3] A. Eremenko, A Markov-type inequality for arbitrary plane continua, Proc. Am. Math. Soc. 135 (5) (2007) $1505-1510$.

[4] H. Gonska, D. Kacsó, I. Raşa, On genuine Bernstein-Durrmeyer operators, Results Math. 50 (3-4) (2007) $213-225$

[5] H. Gonska, P. Piţul, I. Raşa, General king-type operators, Schriftenreiche des Fachbereichs Mathematik, Universdity Dusiburg Essen, SM-DU-665, 2008.

[6] X. Jiang, L. Xie, Simultaneous approximation by Bernstein-Sikkema operators, Anal. Theory Appl. 24 (3) (2008) $237-246$.

[7] I.Ya. Novikov, Asymptotics of the roots of Bernstein polynomials used in the construction of modified Daubechies wavelets, Math. Notes 71 (1-2) (2002) 217-229.

[8] S. Petrone, Random Bernstein polynomials, Scand. J. Stat. 26 (3) (1999) 373-393.

[9] A. Lupaş, A q-analogue of the Bernstein operator, in: University of Cluj-Napoca, Seminar on Numerical and Statistical Calculus, University "BabeşBolyai, Cluj-Napoca, 1987, pp. 85-92, Preprint 87-9.

[10] O. Agratini, On certain $q$-analogues of the Bernstein operators, Carpathian J. Math. 20 (1) (2008) 1-6.

[11] S. Ostrovska, The first decade of the $q$-Bernstein polynomials: results and perspectives, J. of Math. Anal. Approx. Theory 2 (1) (2007) 35-51.

[12] S. Ostrovska, The approximation of power function by the $q$-Bernstein polynomials in the case $q>1$, Math. Inequal. Appl. 11 (3) (2008) $585-597$.

[13] Z. Wu, The saturation of convergence on the interval [0,1] for the $q$-Bernstein polynomials in the case $q>1$, J. Math. Anal. Appl. 357 (1) (2009) 137141.

[14] H. Wang, S. Ostrovska, The norm estimates for the $q$-Bernstein operator in the case $q>1$, Math. Comput. 79 (2010) $353-363$.

[15] V.S. Videnskii, On some classes of $q$-parametric positive operators, Oper. Theory Adv. Appl. 158 (2005) $213-222$.

[16] A. Il'inskii, S. Ostrovska, Convergence of generalized Bernstein polynomials, J. Approx. Theory 116 (2002) 100-112.

[17] Heping Wang, Korovkin-type theorem and application, J. Approx. Theory 132 (2) (2005) $258-264$.

[18] S. Ostrovska, q-Bernstein polynomials of the Cauchy kernel, Appl. Math. Comput. 198 (1) (2008) 261-270.

[19] S. Ostrovska, q-Bernstein polynomials and their iterates, J. Approx. Theory 123 (2003) 232-255.

[20] S. Ostrovska, On the approximation of analytic functions by the $q$-Bernstein polynomials in the case $q>1$, Electron. Trans. Numer. Anal. 37 (2010) $105-$ 112.

[21] S. Cooper, S. Waldron, The eigenstructure of the Bernstein operator, J. Approx. Theory 105 (2000) 133-165.

[22] G.G. Lorentz, Bernstein Polynomials, Chelsea, New York, 1986.

[23] R. Nevanlinna, Analytic Functions, Springer-Verlag, 1970. 